Artigo de Revisão

\title{
Os Agonistas Dopaminérgicos e a Qualidade de Vida na Doença de Parkinson
}

\section{Dopaminergic Agonists and Quality of Life in Parkinson's Disease}

Vitor Tumas ${ }^{1}$

\section{RESUMO}

A doença de Parkinson é uma doença neurodegenerativa em que os sintomas motores predominantes são resultado da morte das células da via nigro-estriatal e conseqüente depleção dopaminérgica no estriado. Os agonistas dopaminérgicos são drogas que atuam diretamente nos receptores de dopamina e sua utilização terapêutica está associada a uma menor freqüência de flutuações motoras em relação ao tratamento com levodopa. O complexo tratamento dessa doença deve ter como um dos objetivos a melhora na qualidade de vida dos pacientes. Os agonistas dopaminérgicos produzem efeito positivo sobre essa variável, possivelmente por diminuírem as complicações do tratamento e possivelmente aliviarem os sintomas depressivos.

\section{Unitermos: Doença de Parkinson; Qualidade de Vida; Agonistas dopaminérgicos; Revisão.}

Citação: Tumas V. Os Agonistas Dopaminérgicos e a Qualidade de Vida na Doença de Parkinson. Rev Neurocienc 2006; 14(2):054-056.

\section{SUMMARY}

The Parkinson's disease is a neurodegenerative condition in which the most common motor symptoms result from the nigrostriatal path cells death and the resulting dopaminergic depletion on striatum. Dopaminergic agonists are drugs acting directly on dopamine receptors and its therapeutic use is associated to a lower frequency of motor fluctuations compared to the treatment with levodopa. The complex treatment of this condition should be targeted to the enhancement of patients' quality of life. Dopaminergic agonists produce a positive effect on this variable, potentially for reducing treatment complications and for providing relief of depressive symptoms.

Keywords: Parkinson disease; Quality of Life; Dopamine agonists; Review.

Citation: Tumas V. Dopaminergic Agonists and Quality of Life in Parkinson's Disease. Rev Neurocienc 2006;14(2):054-056.

\section{INTRODUÇÃO}

A doença de Parkinson (DP) é uma patologia neurodegenerativa caracterizada por uma acentuada perda neuronal na via dopaminérgica nigro-estriatal que provoca sintomas motores característicos e muito incapacitantes. Os pacientes acometidos sofrem sérias limitações funcionais na motricidade, mas também são afligidos por inúmeros outros problemas que da mesma maneira são capazes de produzir um sem fim de dificuldades. Apesar de dispormos atualmente de inúmeros recursos terapêuticos existe ainda muita incerteza sobre a relevância de certas estratégias terapêuticas sobre o bem-estar do paciente. Embora certas medicações possam melhorar evidentemente alguns sintomas dos pacientes é importante determinar seu verdadeiro impacto em aspectos funcionais relevantes. Sendo assim, o objetivo dessa revisão foi descrever alguns aspectos básicos relacionados ao uso de agonistas dopaminérgicos e seu possível impacto na qualidade de vida quando utilizados para o tratamento de pacientes com DP. Para isso, fez-se uma pesquisa na literatura médica procurando estudos que verificaram especificamente o impacto desses medicamentos na qualidade de vida desses pacientes.

\section{OS AGONISTAS DOPAMINÉRGICOS}

A doença de Parkinson (DP) é uma patologia neurodegenerativa caracterizada pela perda de células dopaminérgicas na pars compacta da substância nigra e pela redução significativa na concentração de dopamina no estriado. Os sintomas motores da doença são os que mais se correlacionam com esse déficit e incluem a presença de bradicinesia, rigidez muscular, tremor e instabilidade postural. A DP é na verdade uma patologia com envolvimento multisistêmico, e vários outros sistemas de neurotransmissão são também afetados, porém em menor magnitude que o sistema dopaminérgico. A degeneração de outros grupos neuronais como os do locus ceruleous, núcleos basalis de Meynert, núcleo dorsal do vago, outras vias de projeção meso-corticais, bulbo olfatório, cadeia simpática e até neurônios corticais são responsáveis pelo aparecimento de sintomas não-motores como déficit cognitivo, distúrbios do sono, sintomas autonômicos, sintomas afetivos, etc.

A maioria das drogas utilizadas para o tratamento dos sintomas motores da DP se baseia na estratégia de aumentar a estimulação dopaminérgica no estriado. Isso pode ser

Trabalho realizado na Faculdade de Medicina de Ribeirão Preto da Universidade de São Paulo

1. Professor do Departamento de Neurologia, Psiquiatria e Psicologia Médica da Faculdade de Medicina de Ribeirão Preto da Universidade de São Paulo.

Endereço para correspondência: prof. Dr. Vitor Tumas - Departamento de Neurologia, Psiquiatria e Psicologia Médica da Faculdade de Medicina de Ribeirão Preto da Universidade de São Paulo Campus Universitário Monte Alegre Ribeirão Preto - SP Brasil cep: 14049-900 E-mail: tumasv@rnp.fmrp.usp.br 
obtido aumentando-se o suprimento local de dopamina com a Levodopa, ou então utilizando drogas que ativam diretamente os receptores dopaminérgicos (agonistas), ou ainda prolongando a permanência da dopamina na fenda sináptica com drogas bloqueadoras da recaptação ou da degradação da dopamina.Os agonistas dopaminérgicos são substâncias que agem estimulando diretamente os receptores dopaminérgicos tipo-D2. Essa ação farmacológica é supostamente a principal responsável pelos efeitos terapêuticos sobre os sintomas motores na DP ${ }^{1}$. Entretanto, os mecanismos de ação e os efeitos clínicos dos agonistas dopaminérgicos não estão completamente esclarecidos. As ações desse grupo de drogas sobre o sistema nervoso central dependem da interação relativa de cada uma delas com os diferentes subtipos de receptores dopaminérgicos descritos. Cada droga em particular possui um perfil peculiar de afinidade pelos receptores D1-D2-D3-D4-D5. As diferenças nesse perfil farmacológico são as responsáveis pelas variações nos efeitos terapêuticos e também nos efeitos adversos observados durante o uso das diferentes moléculas terapêuticas.

\section{As aparentes vantagens dos agonistas seriam:}

- eles ativam diretamente os receptores sem a mediação da dopamina, por isso sua ação não depende de qualquer conversão bioquímica no sistema nervoso e sua ação farmacológica independeria do grau de lesão da via nigro-estriatal,

- eles ultrapassam a barreira hemato-encefálica com facilidade e não sofrem concorrência pelo transporte com aminoácidos aromáticos como a Levodopa, assim sua ação clínica seria mais previsível e menos variável.

- seu metabolismo não produz metabólitos tóxicos nem radicais livres, por isso teoricamente não haveria o risco de induzirem uma aceleração local no processo neurodegenerativo,

- a maioria dos agonistas, exceto a Apomorfina, têm meiavida muito mais longa que a Levodopa, assim teriam a capacidade de estimular de maneira mais contínua os receptores dopaminérgicos, não propiciando assim a pulsatilidade na estimulação dopaminérgica.

Como seria esperado prever nesse contexto e como vieram demonstrar vários estudos, podemos afirmar que o uso de agonistas dopaminérgicos está associado a um menor risco de aparecimento das complicações motoras durante o tratamento ${ }^{2,3}$. Muito raramente um paciente em monoterapia com um agonista desenvolve discinesias ou fenômenos "on-off". Porém, seus efeitos clínicos são inferiores aos da Levodopa em todos os estudos comparativos e em qualquer fase da doença. Os principais efeitos práticos da associação de um agonista a um tratamento em curso com a Levodopa são: a redução nos períodos "off" e nas discinesias em médio prazo e a possibilidade de se reduzir a dose terapêutica de Levodopa. Quanto ao seu possível efeito neuroprotetor, demonstrado em vários protocolos experimentais, não há comprovação clínica. Alguns estudos mostraram que o tratamento com um agonista dopaminérgico está associado a uma redução na taxa de queda da captação de marcadores radioativos associadas à transmissão dopaminérgica no estriado. Entretanto, essa observação neuroquímica não se correlacionou com os dados clínicos que não evidenciaram uma desaceleração na progressão dos sintomas ${ }^{4,5}$. Sendo assim, não se pode- afirmar que os agonistas dopaminérgicos tenham um efeito neuroprotetor. Nesse cenário, persiste a discussão entre os especialistas sobre a indicação clínica dos agonistas dopaminérgicos ${ }^{1,2}$. Alguns sugerem que um agonista deve ser a droga de $1^{a}$ escolha no tratamento dos pacientes com DP, especialmente por atrasar a necessidade do uso da Levodopa e o aparecimento das complicações motoras. Muitos concordam com essa visão quando o dilema é iniciar o tratamento de um paciente em que a doença se iniciou em uma idade precoce. Outros apenas concordam em associar um agonista ao tratamento com Levodopa quando começam a aparecer as complicações motoras. Quando os agonistas são utilizados em monoterapia eles produzem um efeito sintomático moderado. À partir de um certo momento eles são incapazes de controlar por si só os sintomas motores, sendo necessário associar a Levodopa ao esquema terapêutico. Essa discussão é também muito motivada por aspectos farmacoeconômicos ligados ao elevado custo relativo dessas drogas. Os agonistas dopaminérgicos utilizados na prática clínica são divididos em ergolínicos e não-ergolínicos. No mercado brasileiro estão disponíveis os agonistas ergolínicos: bromocriptina, cabergolina e lisuride, e os não-ergolínicos: pramipexol, ropinirole e piribedil. Não há estudos comparativos suficientes que esclareçam com precisão as vantagens e desvantagens de cada uma dessas drogas. Aparentemente, a eficácia sintomática é similar quando utilizamos doses terapêuticas equivalentes. Os principais efeitos secundários dos agonistas dopaminérgicos são: náusea, vômitos, tonturas, sonolência, edema, hipotensão ortostática e alucinações. O início lento e gradual da medicação até atingir a dose terapêutica é fundamental para aumentar a tolerância dos pacientes. Efeitos colaterais mais graves como fibrose pleural e retroperitoneal e valvulopatia cardíaca foram descritos durante o uso dos agonistas ergolínicos, especialmente o pergolide ${ }^{6}$. Todas essas considerações devem estar na mente de um clínico quando ele se depara na prática com uma situação em que ele considera a possibilidade de prescrever um agonista dopaminérgico ao seu paciente. Além disso, o rápido avanço no conhecimento científico e as mudanças no modo de encarar e abordar os problemas de saúde fazem com que mais aspectos sejam incluídos nesse organograma que estabelece as vantagens de se escolher determinadas estratégias terapêuticas. O impacto do tratamento sobre a qualidade de vida do paciente é um dos mais importantes fatores considerados atualmente.

\section{QUALIDADE DE VIDA}

Qualidade de vida é um conceito complexo e muito amplo, e de certa forma subjetivo, que mede a sensação de bem-estar e a satisfação do indivíduo com a sua vida. Essa medida tornou-se um item indispensável na avaliação de intervenções terapêuticas em pacientes portadores de doenças crônicas. Há um consenso geral de que é preciso desenvolver terapias que não apenas atenuem sintomas específicos, mas que melhorem a qualidade de vida dos doentes. Para medir essa variável foram desenvolvidos questionários genéricos ou específicos que fazem uma estimativa objetiva da qualidade de vida. Para avaliação de pacientes portadores da DP existem alguns instrumentos específicos como o PDQL 7 , o PIS ${ }^{8}$ e o PDQ3999. Esse último 
é o questionário mais utilizado nos estudos clínicos. Esses instrumentos específicos são mais sensíveis em detectar mudanças no estado de saúde dos pacientes, mas não servem para comparações entre pacientes com múltiplas morbidades ou entre diferentes doenças crônicas. Para essas finalidades é necessário utilizar questionários genéricos. No caso dos pacientes com DP, essa sensação de bem-estar e satisfação com a saúde não é captada pelas escalas clínicas rotineiramente utilizadas como a UPDRS. Além disso, a percepção geral de bem estar nesses pacientes é muito mais determinada por sintomas mentais, como ansiedade e depressão, que pelos sintomas físicos ${ }^{10}$. Embora esses instrumentos sejam cada vez mais utilizados nos estudos terapêuticos recentes, pouco se sabe acerca dos efeitos diferenciais das diferentes medicações antiparkinsonianas sobre essa variável. Os estudos sobre qualidade de vida na DP mostram de maneira contundente que a depressão é um dos fatores negativos mais relevantes ${ }^{11}$. Assim, um dos objetivos a ser obsessivamente perseguido durante o acompanhamento desses pacientes é reconhecer e tratar adequadamente esse problema. Outros fatores importantes associados à queda na qualidade de vida dos pacientes são: a presença de dependência nas atividades do diaa-dia, o uso de doses elevadas de levodopa, a presença distúrbios do sono e flutuações motoras ${ }^{11-13}$. A maioria dos estudos mostra que a qualidade de vida dos pacientes declina com a progressão da doença e está evidentemente associada ao estadio da doença.

\section{TRATAMENTO COM AGONISTAS DOPAMINÉRGI- COS E SEUS EFEITOS SOBRE A QUALIDADE DE VIDA DOS PACIENTES}

Considerando os principais fatores responsáveis pela queda na qualidade de vida dos pacientes, podemos imediatamente identificar vários deles que poderiam ser atenuados pelos efeitos do tratamento com um agonista dopaminérgico, como a redução nas doses terapêuticas da Levodopa, nas flutuações motoras e na dependência funcional. Além disso, existem evidências clínicas de que alguns agonistas dopaminérgicos tenham um efeito antidepressivo. Essa seria uma vantagem significativa dessas medicações já que nenhuma outra droga antiparkinsoniana teria um efeito semelhante sobre esse importante problema. Entretanto, como os agonistas dopaminérgicos atenuam vários sintomas motores e as

\section{REFERÊNCIAS BIBLIOGRÁFICAS}

1. Lees A. Alternatives to levodopa in the initial treatment of early Parkinson's disease. Drugs Aging 2005; 22: 731-740.

2. Clarke CE, Guttman M. Dopaine agonist monotherapy in Parkinson's disease Lancet 2002; 360:1767-1769.

3. Holloway RG, Shoulson I, Fahn S, Kieburtz K, Lang A, Marek K, et al. Pramipexole versus levodopa as initial treatment for Parkinson disease: a 4-year randomized controlled trial. Arch Neurol 2004; 61:1044-1053.

4. Parkinson Study Group. A randomized controlled trial comparing pramipexole with levodopa in early Parkinson's disease: design and methods of the CALM PD Study. Parkinson Study Group. Clin Neuropharmacol 2000; 23:34-44

5. Whone Al, Watts RI, Stoessl Aj, Davis M, Reske S, Nahmias C, et al. Slower progression of Parkinson's disease with ropinirole versus levodopa: The REAL-PET study. Ann Neurol. 2003; 54:93-101.

6. Zadikoff C, Rochon P, Lang A. Cardiac valvulopathy associated with pergolide use. Can J Neurol Sci 2006; 33:27-33.

7. De Boer AG, Wijker W, Speelman JD, De Haes JC. Quality of life in patients with Parkinson's disease: development of a questionnaire. J Neurol Neurosurg Psychiatry 1996, 61:70-74

8. Calne SM, Mak E, Hall J, Fortin MJ, King P, Mclnnes G, et al. Validating a quality of life rating scale for idiopathic parkinsonism: Parkinson's Impact Scale (PIMS). Parkinsonism Relat Dis 1996; 2:55-61.

9. Peto V, Jenkinson C, Fitzpatrick R, Greenhall R. The development and validation of a short measure of functioning and well-being for individuals with complicações motoras, seria válido considerar que o efeito sobre o humor poderia ser apenas resultado da melhora clínica dos pacientes. Um estudo mostrou que o tratamento com Pramipexol de um grupo de pacientes não-flutuantes com depressão maior foi mais eficiente que a prescrição de doses intermediárias a reduzidas de Sertralina ${ }^{14}$. O mesmo efeito antidepressivo foi observado em tratamentos realizados com Ropinirole ${ }^{15}$. Nesses estudos, o efeito antidepressivo não parecia associado ao seu efeito sintomático sobre os sintomas motores. Existem assim inúmeras indicações ainda não totalmente comprovadas de que os agonistas não-ergolínicos poderiam ter um efeito antidepressivo clinicamente significativo. Esse seria um ponto extremamente favorável à utilização desse grupo de drogas quando se pretende melhorar a qualidade de vida dos pacientes com DP. Analisando os resultados de um estudo comparativo entre a Levodopa e o Pramipexol com pacientes acompanhados por 4 anos $^{3}$, Noyes et al. ${ }^{16}$ observaram que as 2 drogas produzem um efeito distinto sobre a qualidade de vida. O efeitos do Pramipexol foram mais evidentes após 3 a 4 anos de tratamento, enquanto nos primeiros anos os efeitos das duas drogas foram equivalentes. Nesse estudo, os efeitos colaterais mais comuns dos agonistas não causaram impacto negativo na qualidade de vida. Essas observações sugerem uma vantagem em longo prazo dos agonistas sobre a Levodopa. Entretanto, não existem estudos comparativos bem conduzidos que possam afirmar que um determinado tipo de medicamento ou estratégia terapêutica seja superior a outra quanto aos seus efeitos positivos sobre a qualidade de vida dos pacientes. Sem dúvida, pela importância que assume a Levodopa no tratamento desses pacientes o ideal seriam estudos que comparassem o seu efeito com o de outras intervenções terapêuticas.

\section{CONCLUSÃO}

Os agonistas dopaminérgicos são drogas importantes no tratamento da DP e parecem produzir efeitos positivos sobre a qualidade de vida dos pacientes. Os estudos disponíveis são ainda muito limitados e são necessários mais estudos comparativos que investiguem o impacto de diferentes medicações e estratégias terapêuticas. Sendo assim, neste momento não é possível afirmar que determinado medicamento antiparkinsoniano é superior a outro nesse quesito funcional complexo.

Parkinson's disease. Qual Life Res 1995; 4: 241-248.

10. Chrischilles EA, Rubenstein LM, Voelker MD, Wallace RB, Rodnitzky RL. Linking clinical variables to health-related quality of life in Parkinson's disease. Parkinsonism Relat Dis 2002; 8:199-209.

11. Sławek J, Derejko M, Lass P. Factors affecting the quality of life of patients with idiopathic Parkinson's disease-a cross-sectional study in an outpatient clinic attendees. Parkinsonism Relat Dis 2005; 11465-11468.

12. Happe S, Berger K, FAQT Study Investigators. The association of dopamine agonists with daytime sleepiness, sleep problems and quality of life in patients with Parkinson's disease--a prospective study. J Neurol 2001 248:1062-1067.

13. Behari M, Srivastava Ak, Pandey RM. Quality of life in patients with Parkinson's disease. Parkinsonism Relat Dis 2005;11:221-226.

14. Barone P, Scarzella L, Marconi R, Antonini A, Morgante L, Bracco F, et al. Pramipexole versus sertraline in the treatment of depression in Parkinson's disease: A national multicenter parallel-group randomized study. J Neurol 2006; 253:601-607

15. Cassano P, Lattanzi L, Fava M, Navari S, Battistini G, Abelli M, et al. Ropinirole in treatment-resistant depression: a 16-week pilot study. Can J Psychiatry 2005; 50:357-360.

16. Noyes K, Dick AW, Holloway RG, The Parkinson Study Group. Pramipexole versus levodopa in patients with early Parkinson's disease: effect on generic and disease-specific quality of life. Value Health 2006;9:28-38. 